\title{
Bending Nanowire Growth in Solution by Mechanical Disturbance
}

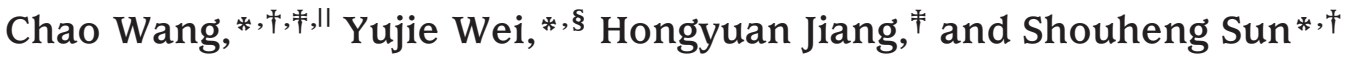 \\ ${ }^{\dagger}$ Department of Chemistry and ${ }^{*}$ Division of Engineering, Brown University, Providence, Rhode Island 02912 and \\ $\S$ State Key Laboratory of Nonlinear Mechanics, Chinese Academy of Sciences, Beijing, 100190, People’s Republic of China
}

\begin{abstract}
The effect of mechanical disturbance on one-dimensional nanocrystal growth in solution phase is investigated by controlled growth of Au nanowires with and without stirring. While a static growth leads to straight, single-crystal Au nanowires, the mechanic disturbance by stirring tends to bend the nanowire growth, yielding nanowire kinks abundant in various types of crystal defects including dislocations, twin boundaries, and grain boundaries. Mechanical modeling and analysis is introduced to elucidate the nanowire growth mechanisms in these two conditions. The provided fundamental understanding of crystal defect formation at nanoscale could be applied to guide the development of advanced nanomaterials with shape control and unique mechanical properties.
\end{abstract}

KEYWORDS Gold nanowires, solution synthesis, bending nanowire growth, mechanical disturbance, nanocrystal defect, reverse micelle

S hape-controlled synthesis of nanomaterials with chemical and physical properties tailored by the material morphology has long been pursued. ${ }^{1-4}$ In this context, the approach with nanocrystal growth and process in solution phase has been demonstrated to be promising, and nanocrystal shape control in sphere, ${ }^{5}$ cube, $^{6}$ polyhedron, ${ }^{7}$ nanorod, ${ }^{8,9}$ or nanowire (NW) ${ }^{10-13}$ has been achieved. ${ }^{14,15}$ Many factors including thermodynamic energetics, ${ }^{2}$ reaction and growth kinetics, ${ }^{16}$ surface ligand selectivity, ${ }^{17}$ seeding ${ }^{15,18}$ and template ${ }^{19}$ are proposed to explain the nanocrystal growth into diversified morphologies. Ultrathin (<10 nm) Au NWs were recently synthesized in organic solution with the assistance of the reverse-micelle structure formed by oleylamine. ${ }^{11-13}$ Despite the chemical reaction mechanisms studied therein, we recently reported that mechanical disturbance could have a substantial effect on the one-dimensional (1D) nanocrystal growth, where ultrathin single-crystal NWs of micrometers long can only be obtained without stirring. ${ }^{12}$ This has inspired the further investigation of the potential role of mechanical disturbance in controlled nanomaterial synthesis.

While the ultrathin, defect-free Au NWs show great potential as interconnectors for nanoelectronic devices and circuits, ${ }^{12}$ on the other hand we note that the presence of defects in NWs may not be disadvantageous for other applications. Twin and grain boundaries are generally considered to be deleterious to the electrical and optical properties of materials, whereas recent molecular-dynamics simulations have revealed that the spaced twins in NWs can block the propagation of dislocation in certain crystallographic

\footnotetext{
*To whom correspondence should be addressed. E-mail: (C.W.) chaowang@ anl.gov; (Y.W.) yujie_wei@Inm.imech.ac.cn; (S.S.) ssun@brown.edu.

"Current Address: Materials Science Division, Argonne National Laboratory, Argonne, IL 60439.

Received for review: 02/23/2010

Published on Web: 05/25/2010
}

orientations and hence dramatically enhance the NW strength. ${ }^{20-24}$ Experimentally, axial screw dislocation has been observed to drive the vapor-liquid-solid growth of semiconductor NWs into pine treelike hierarchical nanostructures, which is believed to have great potential in harvesting solar energy. ${ }^{25}$ Methodologies to bring the dense twin boundaries into bulk polycrystalline materials have also been explored. ${ }^{26-28}$ Using electric deposition, hierarchy structure of nanotwinned polycrystalline $\mathrm{Cu}$ has been obtained, which shows high mechanical strength of $\sim 1 \mathrm{GPa}$ and similar electrical conductivity to conventional coarse grained $\mathrm{Cu}^{28-30}$

Here we report the comparative study of growing ultrathin Au NWs in an organic solution with and without mechanical stirring. While a static growth led to straight, single-crystal Au NWs, mechanical stirring disrupted the 1D nanocrystal growth, causing the NWs to bend. Various types of crystal defects including dislocations, twin boundaries, and grain boundaries were observed in the formed NW kinks via high-resolution electron microscopic analysis. Mechanical modeling and analysis on the growth of bent NWs revealed that the formation of these crystal defects was due to the rather low energies of gold face-centered cubic (fcc) crystal stacking faults and for deforming/redistributing oleylamine surfactants in respect of the disturbance by mechanical stirring.

The synthesis route was slightly modified compared to the previous recipe. In a typical synthesis of $9 \mathrm{~nm}$ Au NWs, $0.2 \mathrm{~g}$ of $\mathrm{HAuCl}_{4}$ (Strem) was added to $20 \mathrm{~mL}$ oleylamine $(70 \%)$ at room temperature. Here oleylamine served as the solvent, reducing agent and stabilizer. The formed solution was heated to $80{ }^{\circ} \mathrm{C}$ under vigorous magnetic stirring. Hexane was evaporated under nitrogen flow. The solution was kept at this temperature for $24 \mathrm{~h}$ with or without stirring. The Au NWs were precipitated out by adding ethanol and 



FIGURE 1. TEM images of Au NWs: (a) $3 \mathrm{~nm}$ without stirring, (b) 3 $\mathrm{nm}$ with stirring, (c) $9 \mathrm{~nm}$ without stirring, (d) $9 \mathrm{~nm}$ with stirring.

centrifugation. The dark-red product was washed twice with ethanol and redispersed in hexane.

Figure 1 shows the transmission electron microscopy (TEM) images of Au NWs obtained with and without stirring respectively. Both the 3 and 9 nm NWs grown without the disturbance are found to be straight and uniform of micrometers in length (Figure 1a,b). The product from synthesis with stirring contains a large ration of Au NPs $(8-10 \mathrm{~nm}$, Figure $1 \mathrm{~b}, \mathrm{~d}$ ). In addition, a lot of kinks can be seen, as labeled by dashed circles in Figure $1 \mathrm{~b}, \mathrm{~d}$. In most cases, the bending angle is close to $109.5^{\circ}$, the angle between two sets of (111) planes in fcc materials. Kinks of other angles have also been observed but are not as dominant. Another observation is for the tails of the NWs, which are almost identical hemispheres in NWs grown without stirring, while for NWs obtained with stirring big bumps $(>10 \mathrm{~nm})$ are frequently found at the ends (see the red arrows in both Figure $1 \mathrm{c}, \mathrm{d})$. These observations strongly suggest that the mechanical disturbance has significantly altered the microscopic environment for the nanocrystal growth.

High-resolution TEM (HRTEM) images reveal that multiple types of defects exist in the NWs obtained with stirring in sharp contrast to the single-crystal NWs grown without disturbance. ${ }^{12}$ Figure 2 a shows a typical kink with a onethird [111] Frank partial dislocation ${ }^{31}$ with the inset showing the typical structure by stacking atomic layers in fcc metals. Frank partial dislocation usually forms when one portion of a layer is removed, or alternatively one layer (marked with red line) was only partially deposited before stacking of the next layer. Considering the high-energy level of such a dislocation, it is likely formed by quick deposition of atoms from the peripheral and the growth advancing to the center,
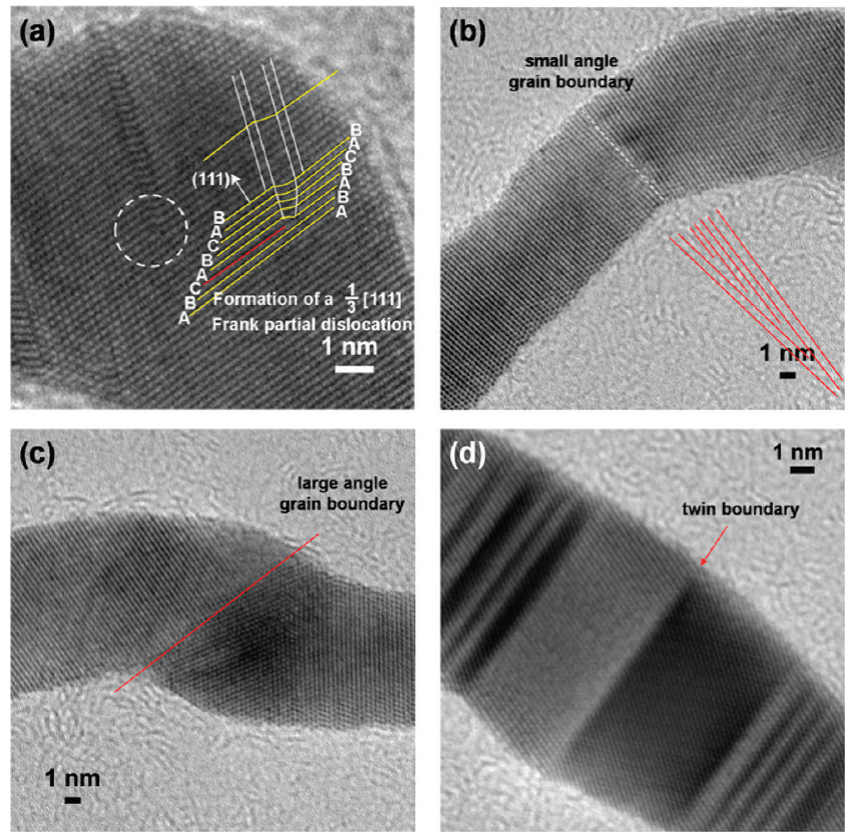

FIGURE 2. HRTEM images of different types of defects observed in NWs grown with mechanical disturbance. (a) A typical kink with a stacking fault and a Frank partial with a sketch illustrating the lattice structure by stacking atomic layers in fcc metals with A, B, and C referring to different stacking layers. The illustration is shifted away from the defect (dashed circle) for clarity. (b) A low-angle grain boundary nearby a kink. (c) A high-angle grain boundary. (d) A segment of dense twin bands.

where the dislocation forms when the fronts of deposition merge at the NW center with a mismatch. This scenario is consistent with previous theoretical analysis ${ }^{32}$ and experimental observations ${ }^{33}$ for semiconductor NWs. A smallangle grain boundary is seen in Figure $2 \mathrm{~b}$, where the growth of (111) plane is slightly tilted. The structure of the smallangle grain boundary resembles an array of dislocations along a line. The energy of this type of defect is usually considered to be proportional to the tilted angle and rather low, ${ }^{34}$ which mechanical disturbance could be sufficient to induce. Despite that, large-angle grain boundary does exist in the NWs grown in the presence of mechanical disturbance, as shown in Figure $2 \mathrm{c}$ where the growth direction is severely altered. The formation mechanism of such highenergy grain boundary is yet unclear for NWs growth in solution, but it may result from wire-wire or wire-particle fusion. ${ }^{35}$ Excessive twins and stacking faults are also seen in NWs obtained under stirring, as shown in Figure 2d, where the pattern of alternative gray and dark stripes is caused by the different fringe orientation between two successive segments. Such type of defect has been broadly observed in semiconductor NWs. ${ }^{36-39}$

Particularly, the NW kink is an interesting structure in 1D nanocrystal growth. It has been recently reported that kinked and zigzag semiconductor NWs can be synthesized by chemical vapor deposition (CVD) with purging gas stream ${ }^{40}$ and the obtained nanostructures show great potential of application in nanoscale electronics and biotech- 

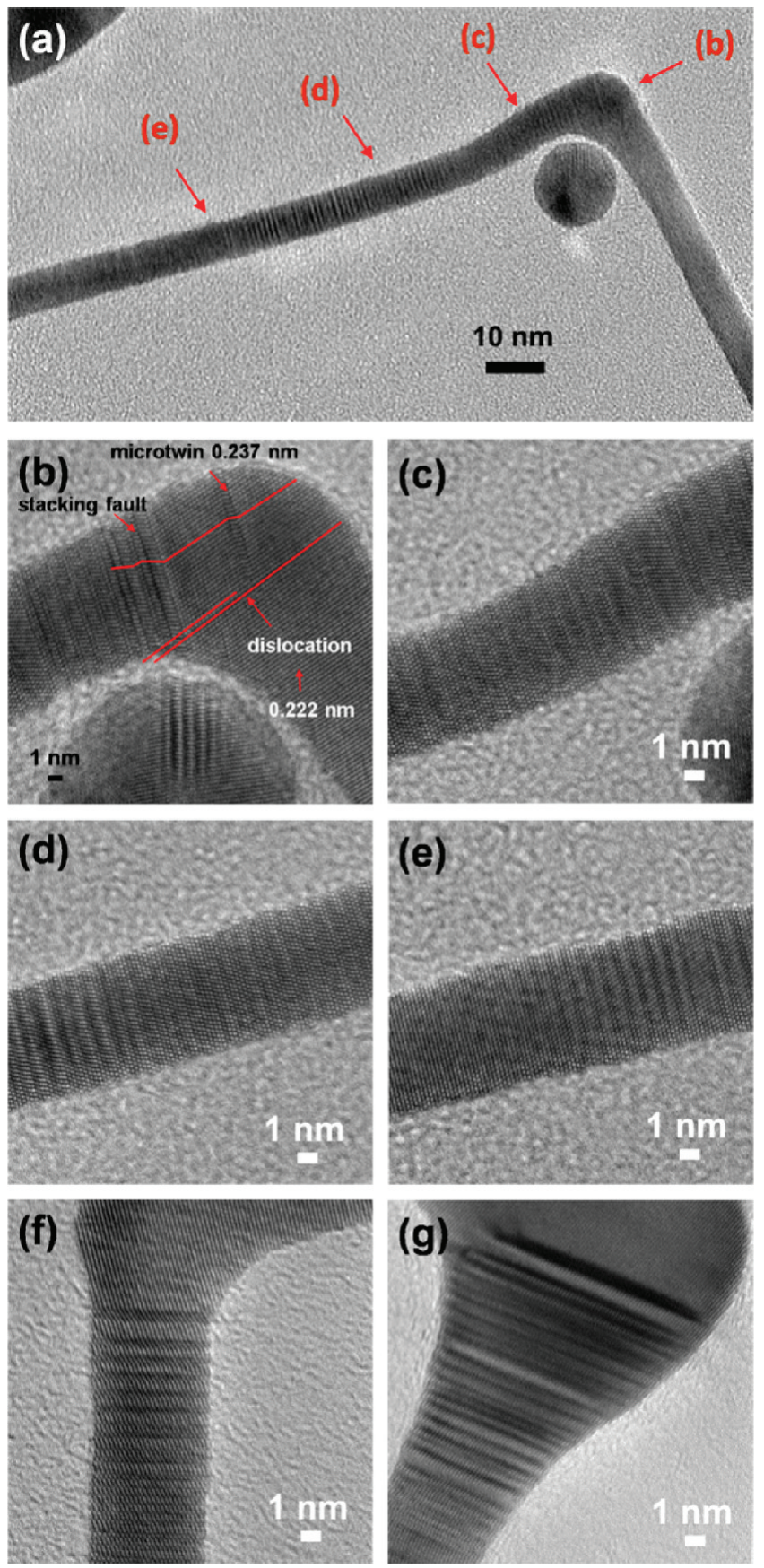

FIGURE 3. HRTEM images of a representative Au NW kink: (a) overview and $(b-e)$ segments at different positions along the NW as labeled in (a). (f,g) HRTEM images of another two similar kinks.

nology. ${ }^{41}$ While the formation of NW kinks in the oriented anisotropic CVD growth is somehow within expectation, it is quite surprising to obtain such nanostructures from a homogeneous organic solution synthesis. Figure 3 shows the HRTEM images of a typical kink structure observed for NWs grown with stirring. The overview image (Figure 3a) reveals that the bending angle is about $110^{\circ}$, consistent with the above observation from low-magnification TEM image (Figure 1d). Magnified images of the successive segments (a)

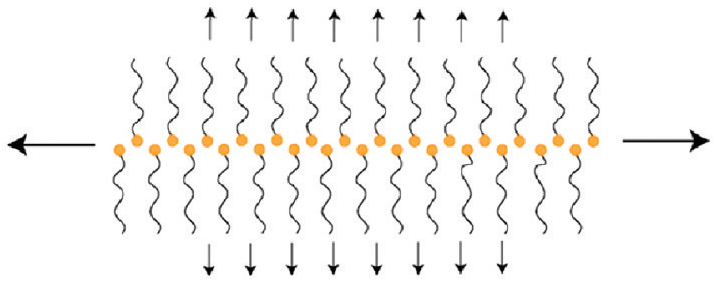

(b)

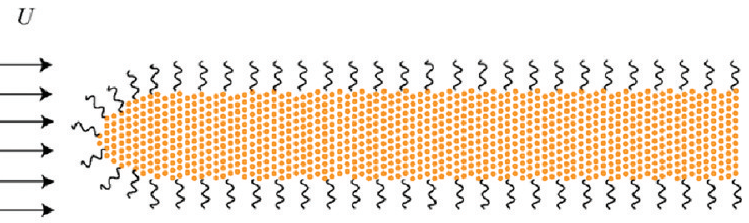

(c)

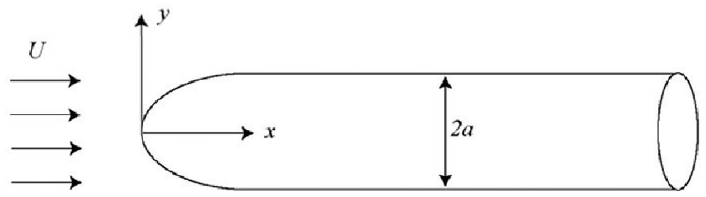

FIGURE 4. Schematic illustration of the growth of Au NWs and the influence of stirring. (a) The backbone structure formed by aurophilic bonding at the early stage of NW growth. Axial and radial growths are respectively indicated by arrows. (b) Illustration of NWs covered by oleylamine layer in a fluid with far field velocity $U$. (c) The idealized model of NW growth with a tipped cylinder (radius $a$ ) in a viscous flow with axial velocity $U$.

labeled in Figure $3 a$ are respectively shown in Figure 3b-e. The NW shown in Figure $3 a$ is believed to grow from the right-hand side, since almost no defect can be seen for this segment before the appearance of kink (also see the right part of the wire shown in Figure 3b). Accompanying the change of growth direction, extensive crystal defects appear, including a partial dislocation (Figure 3b, also detailed in Figure 2a), dense stacking faults and twin boundaries with typical spacing no more than $1 \mathrm{~nm}$ (Figure $3 \mathrm{~b}-\mathrm{d}$ ). Note that in Figure 3b (left-hand side), those sharp black lines appearing between two successive segments of lattice fringes that have coherent crystalline orientation are resulted from stacking faults. The kink seems to influence the subsequent growth of NW in a length of about tens of nanometers, after which the stacking faults are reduced dramatically and the wire retrieves its single-crystal nature (Figure 3e). For most bent NWs, the crystal pattern of kink structure is quite similar (Figure 3f,g), where they emerge from single-crystalline wires, turn around at the kink, undergo a fragment with coexisting twins and stacking faults, and finally resume their normal, defect-free growth.

Further insights into the above experimental observations are provided by a qualitative model of the defect formation in the Au NWs during their growth subjected to mechanical disturbance. In the absence of disturbance, the Au-oleylamine complex will form a 1D chain structure via the aurophilic bonding between Au atoms, ${ }^{42-44}$ as shown in Figure $4 a{ }^{11,13}$ The wire nucleus in static solution is able to grow in both axial and radial directions. In this case, the NW surface morphology is governed by the reverse micelle 
structure composed of amphiphilic oleylamine molecules instead of Au crystal surface energy. ${ }^{12,13}$ The deposition of Au atoms in the axial direction is usually faster than that in the radial direction (Figure $4 \mathrm{~b}$ ) as the dense molecule wall of oleylamine hinders the diffusion and accumulation of $\mathrm{Au}$ species, leading to the elongated growth into NW.

The presence of stirring breaks the equilibrium of NW growth in static solution. An immediate consequence of this is the breaking of many $1 \mathrm{D}$ Au-oleylamine structures into small segments. ${ }^{11-13}$ A mathematical description of this transition would be challenging, but apparently the interruption of the anisotropic growth by stirring will lead to the formation of spherical nanoparticles (Figure 1b,d). In the following simulation study, we would rather focus on how the NW growth was bent and the formation of crystal defects was induced by the mechanical disturbance. Given the formation of the 1D Au-oleylamine nanostructures, the continuing growth into NWs in the stirred viscous solution is subject to shearing stress imposed by the fluid. An idealized system is introduced to estimate the level of stress applied to NW surface under stirring, as shown in Figure 4c. We set the origin of the coordinate at the tip of a NW with radius $a$. A uniform velocity of $U$ along the wire axial direction is assumed in the viscous solution under stirring. Shear stress nearby the tip of the wire can thus be approximated to be ${ }^{45,46}$

$$
\tau_{0} \approx \frac{\mu U}{a}
$$

where $\mu$ is the viscosity of the fluid. The shear stress at some distance away from the origin $(x \gg a)$ is given as $^{47}$

$$
\begin{array}{r}
\frac{\tau a}{\mu U}=\frac{2}{\beta}+\frac{2 \gamma}{\beta^{2}}+\frac{2\left(\gamma^{2}-\pi^{2} / 4-2 \ln 2\right)}{\beta^{3}}+ \\
o\left(\frac{1}{\beta^{4}}\right) \text { with } \beta=\ln \left(4 \frac{x}{a} / \frac{U a}{v}\right)
\end{array}
$$

Here $\gamma$ is the Euler constant and $v$ the kinematic viscosity, with $v=\mu / \rho$ for $\rho$ being the fluid density. Given the high ratio of length over radius (hence increasing boundary layer as $x$ increases), shear stress on the surface of the wire certain distance away from the head/tail of a NW is only about one tenth of that at its head. The viscosity $\mu$ of the solution is in the region of $10^{-2}$ to $1 \mathrm{pa} \cdot \mathrm{s}$ and the velocity by the stirring is about $10^{-2} \mathrm{~m} / \mathrm{s}(600 \mathrm{rpm}, 1$ in. magnetic bar). The estimated shear stress at the head of a NW is $\tau=0.2-5 \mathrm{MPa}$ for NWs with the radii $a \sim 5 \mathrm{~nm}$. The shear stress in the middle of wires depends on the exact location, but usually is about 1 order of magnitude smaller. As revealed by Bachman et al., for $n$-alkylisonitrile complex of gold(I) chloride $^{44}$ the outer layer formed by long alkyl chains is me-
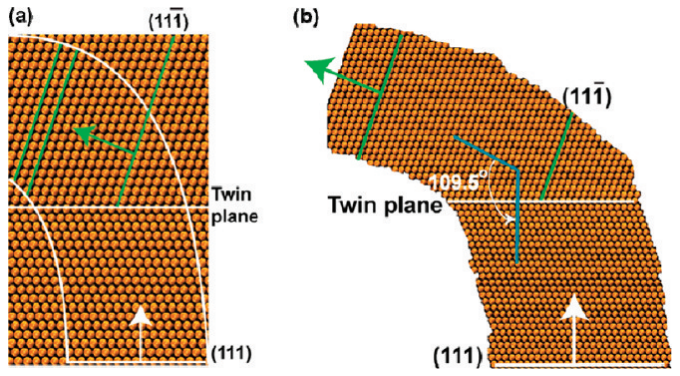

FIGURE 5. Illustration of the formation of kinks in NWs whose growth were disturbed by stirring. (a) The crystallographic structure of a fcc matrix wire growing in $\langle 111\rangle$ direction with a twin plane. (b) Because of the twin plane and probable nonuniform distribution of oleylamine wall induced by viscous flow, the preferential deposition plane turns from (111) to (11) $)$. Note that the angle between these two set of planes is $109.5^{\circ}$.

chanically soft and could be deformed by gentle stresses. Considering that the long stretched hydrocarbon chains of oleylamine is similar to the alkyl chains in their system, it is therefore expected that shear stress produced by stirring in the viscous solution is sufficient to reshuffle the location of oleylamine chains on NW surface or even blow them off. The redistribution of oleylamine chains may cause one side of NW unpassivated by the ligands, giving rise to rapid growth along this direction where crystal defects preferentially form. This mechanism could be responsible for the enrichment of various defects in the NWs grown with stirring. Particularly, the high occurrence rate of twins and stacking faults could be attributed to the relative low energy of such defects compared to the shear stress induced by the stirring. For example, twin boundary energy $\left(\gamma_{\mathrm{tw}}\right)$ is about $32 \mathrm{~mJ} / \mathrm{m}^{2}$ in $\mathrm{Au} .{ }^{34}$ Since the formation of this type of defect usually starts from the periphery of a wire, as depicted in Figure 2a, typical stress required for forming such defects can be approximated as $\gamma_{\mathrm{tw}} / a$ with $a$ being the wire radius. Taking $a=5 \mathrm{~nm}$, the shear stress triggering the formation of such defect is about $6 \mathrm{MPa}$, which is in the order of the stress induced by stirring.

While the formation of abundant defects corresponds to the disturbed flow dynamics, the unique feature of NWs grown with stirring, the kink however can be interpreted by a crystallographic structure analysis of the nanocrystal growth. Figure 5 illustrates the observed bending angle of about $110^{\circ}$ could be a collaborative product of the generation of a stacking fault (Figure $5 \mathrm{a}$ ) and the deformation of oleylamine wall induced by viscous flow, which results in fast growth of alternative ( $11 \overline{1}$ ) planes (Figure $5 b$ ). The angle between (111) and ( $11 \overline{1})$ planes is $109.5^{\circ}$, which is consistent with the experimental observations. The appearance of such a kink will modify the shear stress on the wire surface, especially the region nearby the kink, which might induce the highly dense stacking faults as shown in Figure 3.

In summary, we have investigated the effect of mechanical disturbance on Au nanowire growth in solution phase. Bent nanowires enriched by various types of crystal defects, 
including dislocations, twin boundaries, and grain boundaries around the kinks were obtained from growth in the presence of mechanical disturbance, in contrast to the straight, single-crystal nanowires obtained from static solution. With mechanical modeling and analysis of the crystal defects formation at nanoscale, we deduce that their extensive occurrence could be owing to the rather low energy of stacking faults of gold fcc crystal and deformation/redistribution of oleylamine surfactant layer caused by viscous flow under stirring. The obtained nanostructures could be promising candidates for fabrication of novel nanodevices. The developed strategies and fundamental understandings can be generalized to guide the further development of advanced nanomaterials with shape control and/or enhanced mechanical properties.

Acknowledgment. The work was supported by NSF/DMR 0606264, the Brown University Seed Fund, a scholarship from Hitachi Maxell, Ltd. We thank Professor L. Ben Freund and Professor $\mathrm{H}$. Gao of Brown University for valuable discussions. The help on HRTEM from Mr. Anthony McCormick at Materials Research Center of Brown University is also gratefully acknowledged. Y.W. acknowledges the support of "BaiRen Project" from the Chinese Academy of Sciences

\section{REFERENCES AND NOTES}

(1) Burda, C.; Chen, X. B.; Narayanan, R.; El-Sayed, M. A. Chem. Rev. (Washington, DC, U.S.) 2005, 105, 1025.

(2) Cushing, B. L.; Kolesnichenko, V. L.; O'Connor, C. J. Chem. Rev. (Washington, DC, U.S.) 2004, 104, 3893

(3) Jun, Y. W.; Choi, J. S.; Cheon, J. Angew. Chem., Int. Ed. 2006, 45, 3414.

(4) Murray, C. B.; Kagan, C. R.; Bawendi, M. G. Annu. Rev. Mater. Sci. $2000,30,545$

(5) Sun, S. H.; Murray, C. B.; Weller, D.; Folks, L.; Moser, A. Science 2000, 287, 1989

(6) Sun, Y. G.; Xia, Y. N. Science 2002, 298, 2176

(7) Xia, Y.; Xiong, Y. J.; Lim, B.; Skrabalak, S. E. Angew. Chem., Int. Ed. 2009, 48, 60

(8) Gao, J. X.; Bender, C. M.; Murphy, C. J. Langmuir 2003, 19, 9065.

(9) Peng, X. G.; Manna, L.; Yang, W. D.; Wickham, J.; Scher, E.; Kadavanich, A.; Alivisatos, A. P. Nature 2000, 404, 59

(10) Wang, C.; Hou, Y. L.; Kim, J. M.; Sun, S. H. Angew. Chem., Int. Ed. $2007,46,6333$

(11) Lu, X. M.; Yavuz, M. S.; Tuan, H. Y.; Korgel, B. A.; Xia, Y. N. J. Am Chem. Soc. 2008, 130, 8900

(12) Wang, C.; Hu, Y. J.; Lieber, C. M.; Sun, S. H. J. Am. Chem. Soc. $2008,130,8902$
(13) Huo, Z. Y.; Tsung, C. K.; Huang, W. Y.; Zhang, X. F.; Yang, P. D. Nano Lett. 2008, 8, 2041.

(14) Wang, C.; Xu, C. J.; Zeng, H.; Sun, S. H. Adv. Mater. 2009, 21, 3045.

(15) Habas, S. E.; Lee, H.; Radmilovic, V.; Somorjai, G. A.; Yang, P Nat. Mater. 2007, 6, 692.

(16) Ren, J. T.; Tilley, R. D. J. Am. Chem. Soc. 2007, 129, 3287.

(17) Wang, C.; Daimon, H.; Lee, Y.; Kim, J.; Sun, S. J. Am. Chem. Soc. 2007, 129, 6974

(18) Wang, C.; Daimon, H.; Sun, S. H. Nano Lett. 2009, 9, 1493.

(19) Piao, Y. Z.; Kim, H. J. Nanosci. Nanotechnol. 2009, 9, 2215.

(20) Cao, A. J.; Wei, Y. G.J. Appl. Phys. 2007, 102, 83511.

(21) Deng, C.; Sansoz, F. Nano Lett. 2009, 9, 1517.

(22) Li, L.; Ghoniem, N. M. Phys. Rev. B 2009, 79, 75444

(23) Shabib, I.; Miller, R. E. Acta Mater. 2009, 57, 4364.

(24) Li, X. Y.; Wei, Y. J.; Lu, L.; Lu, K.; Gao, H. J. Nature 2010, 464, 877 .

(25) Bierman, M. J.; Lau, Y. K. A.; Kvit, A. V.; Schmitt, A. L.; Jin, S. Science 2008, 320, 1060.

(26) Zhang, X.; Misra, A.; Wang, H.; Shen, T. D.; Nastasi, M.; Mitchell, T. E.; Hirth, J. P.; Hoagland, R. G.; Embury, J. D. Acta Mater. 2004, $52,995$.

(27) Hodge, A. M.; Wang, Y. M.; Barbee, T. W. Mater. Sci. Eng., A 2006, 429, 272 .

(28) Lu, L.; Shen, Y. F.; Chen, X. H.; Qian, L. H.; Lu, K. Science 2004, 304, 422 .

(29) Lu, L.; Chen, X.; Huang, X.; Lu, K. Science 2009, 323, 607.

(30) Lu, K.; Lu, L.; Suresh, S. Science 2009, 324, 349.

(31) Read, W. T. Dislocations in crystals; McGraw-Hill: New York, 1953; p 228

(32) Glas, F.; Harmand, J. C.; Patriarche, G. Phys. Rev. Lett. 2007, 99, 146101 .

(33) Shtrikman, H.; Popovitz-Biro, R.; Kretinin, A.; Heiblumf, M. Nano Lett. 2009, 9, 215.

(34) Hirth, J. P.; Lothe, J. Theory of dislocations, 2nd ed.; Wiley: New York, 1982; p 857

(35) Halder, A.; Ravishankar, N. Adv. Mater. 2007, 19, 1854

(36) Li, Q.; Gong, X. G.; Wang, C. R.; Wang, J.; Ip, K.; Hark, S. Adv. Mater. 2004, 16, 1436.

(37) Gao, P. X.; Ding, Y.; Mai, W. J.; Hughes, W. L.; Lao, C. S.; Wang, Z. L. Science 2005, 309, 1700

(38) Hao, Y. F.; Meng, G. W.; Wang, Z. L.; Ye, C. H.; Zhang, L. D. Nano Lett. 2006, 6, 1650.

(39) Xiong, Q. H.; Wang, J.; Eklund, P. C. Nano Lett. 2006, 6, 2736.

(40) Tian, B.; Xie, P.; Kempa, T. J.; Bell, D. C.; Lieber, C. M. Nat. Nanotechnol. [Online early access]. Doi:10.1038/nnano.2009.304.

(41) Lieber, C. M. Nanowires as a platform for nanoscience and nanotechnology. In The 238th ACS National Meeting, Washington, DC, Aug 16-20, 2009; American Chemical Society: Washington, DC, 2009

(42) Hollatz, C.; Schier, A.; Schmidbaur, H. J. Am. Chem. Soc. 1997, $119,8115$.

(43) Schmidbaur, H. Gold Bull. 2000, 33, 3

(44) Bachman, R. E.; Fioritto, M. S.; Fetics, S. K.; Cocker, T. M. J. Am. Chem. Soc. 2001, 123, 5376.

(45) Blasius, H. Z. Math. Phys. 1908, 56, 1

(46) Sawchuk, S. P.; Zamir, M. Int. J. Heat Fluid Flow 1992, 13, 184

(47) Glauert, M. B.; Lighthill, M. J. Proc. R. Soc. London 1954, 230, 189 